\title{
Estudio por ecoendoscopia de la vía biliar extrahepática en pacientes con pancreatitis aguda biliar
}

\author{
A. Repiso, R. Gómez-Rodríguez, A. García-Vela, M. J. Pérez-Grueso, R. Martín, M. Romero \\ y J. M. Carrobles
}

Servicio de Aparato Digestivo. Hospital Virgen de la Salud. Toledo

\section{RESUMEN}

Objetivo: el objetivo de nuestro estudio fue valorar la utilidad de la ecoendoscopia en el estudio de la vía biliar extrahepática en los pacientes diagnosticados de pancreatitis aguda biliar y determinar los factores clínicos y analíticos relacionados con el resultado de la técnica.

Material y métodos: se incluyeron en el estudio de modo consecutivo 73 pacientes (31 varones, 42 mujeres; media de edad $64 \pm$ 15 años) con pancreatitis aguda biliar remitidos a nuestro servicio para la realización de ecoendoscopia biliopancreática. En todos los pacientes se realizó la técnica seguida de CPRE con esfinterotomía y técnica endoscópica para la extracción de cálculos cuando se identificó por ecoendoscopia la existencia de coledocolitiasis. Se compararon las características clínico-evolutivas de estos pacientes con respecto al resultado obtenido con la ecoendoscopia.

Resultados: la media de tiempo transcurrido desde el ingreso hasta la realización de la ecoendoscopia fue de $7 \pm 6$ días. En 18 pacientes (24\%) se observó en la ecoendoscopia la existencia de coledocolitiasis y en 17 de ellos se realizó esfinterotomía endoscópica. La presencia de coledocolitiasis fue más frecuente en aquellos pacientes con dilatación de la vía biliar extrahepática (55 vs. $14 \% ; p<0,05)$ y en aquellos con bilirrubina sérica superior a 3 $\mathrm{mg} / \mathrm{dl}(41$ vs. $18 \%$; $\mathrm{p}<0,05)$. Trece pacientes (18\%) presentaron pancreatitis aguda severa. Catorce (19\%) presentaron alguna complicación en relación con la pancreatitis aguda y un paciente murió. Se observó una complicación relacionada con la esfinterotomía endoscópica. Cuatro pacientes presentaron un nuevo episodio de pancreatitis aguda. No se observaron diferencias significativas en el porcentaje de complicaciones en los pacientes tratados de modo conservador respecto a aquellos con coledocolitiasis tratados con esfinterotomía endoscópica (18 vs. 22\%; p > 0,05 ). Tampoco se observó esta diferencia en el subgrupo de pacientes con pancreatitis aguda severa ( 45 vs. 55\%; p > 0,05)

Conclusiones: la ecoendoscopia es una técnica útil en la selección de los pacientes con pancreatitis aguda biliar que se beneficiarán de la realización de una esfinterotomía endoscópica.

Palabras clave: Ecoendoscopia. Pancreatitis aguda biliar. Coledocolitiasis.

\begin{abstract}
Objective: the objective of our study was to evaluate the usefulness of endoscopic ultrasonography (EUS) for the study of the common bile duct in patients diagnosed with acute biliary pancreatitis, and to establish clinical and laboratory factors related to this technique.
\end{abstract}

Materials and methods: seventy-three consecutive patients with acute biliary pancreatitis were included in the study (31 males and 42 females with a mean age of $64 \pm 15$ ) who were admitted to our department for biliopancreatic EUS. In all patients the technique was followed by ERCP with sphincterotomy, and endoscopy to remove stones when endoscopy revealed choledocholithiasis.

Results: mean time from admission to echoendoscopy was $7 \pm 6$ days. In 18 patients (24\%) the presence of choledocholithiasis was revealed by EUS, and in 17 a sphincterotomy was performed. Choledocholithiasis was more frequent in patients with common bile duct dilation (55 vs. 14\%; $\mathrm{p}<0.05$ ). Thirteen patients (18\%) showed severe acute pancreatitis. Fourteen (19\%) showed complications related to acute pancreatitis, and one patient died. Four patients had a new episode of acute pancreatitis. No significant difference was seen in the percentage of complications between patients treated conservatively and patients with choledocholithiasis treated with endoscopic sphincterotomy (18 vs. $22 \%$; $p>0.05$ ). No difference was also detected for the subgroup of patients with severe acute pancreatitis (45 vs. $55 \%$; p > 0.05).

Conclusions: EUS is a useful technique for the selection of patients with acute biliary pancreatitis who may benefit from endoscopic sphincterotomy.

Key words: Endoscopic ultrasonography. Acute biliary pancreatitis. Choledocholithiasis. 
Repiso A, Gómez-Rodríguez R, García-Vela A, Pérez-Grueso MJ, Martín R, Romero M, Carrobles JM. Estudio por ecoendoscopia de la vía biliar extrahepática en pacientes con pancreatitis aguda biliar. Rev Esp Enferm Dig 2008; 100: 337-342.

\section{INTRODUCCIÓN}

La litiasis biliar es responsable de la mayor parte de las pancreatitis agudas en los países industrializados (1). Los mecanismos que podrían estar implicados en el inicio del proceso inflamatorio en estos pacientes incluyen el reflujo del contenido biliar al conducto pancreático y la obstrucción del conducto por la impactación de litiasis a nivel papilar (2). Sin embargo, la mayor parte de las coledocolitiasis en estos pacientes son eliminadas espontáneamente transcurridas pocas horas o días del inicio de los síntomas.

La persistencia de coledocolitiasis en estos casos se asocia con una peor evolución clínica y una mayor tasa de complicaciones, pues la colangitis causada por los cálculos retenidos podría ser responsable, al menos en parte, de la sepsis y/o insuficiencia orgánica presente en algunas ocasiones. En estos pacientes con pancreatitis aguda biliar que presentan colangitis aguda y en aquellos con alta sospecha de presentar coledocolitiasis queda demostrado el efecto beneficioso de la esfinterotomía endoscópica (3-5).

Sin embargo, el potencial efecto beneficioso de la esfinterotomía endoscópica en pacientes con pancreatitis aguda en ausencia de colangitis o alta sospecha de coledocolitiasis es controvertida, pues no ha sido claramente establecido si su realización puede aminorar la insuficiencia orgánica en relación con la inflamación pancreática.

La CPRE (colangiografía retrógrada endoscópica) ha sido considerada durante muchos años la técnica de elección en el diagnóstico preoperatorio de la coledocolitiasis. Sin embargo, se asocia con una tasa no despreciable de complicaciones y la información diagnóstica que aporta es limitada (6-8).

La ecoendoscopia es una técnica mínimamente invasiva que permite obtener imágenes de alta calidad del sistema biliopancreático, que podría reemplazar a la CPRE diagnóstica en el estudio de la vía biliar extrahepática $(9,10)$ y que ha sido utilizada con un alto rendimiento diagnóstico en el estudio de la vía biliar extrahepática en pacientes con pancreatitis aguda biliar (11-15) a pesar de las dificultades técnicas que plantearía la exploración de la vía biliar en esta indicación (11). Sin embargo, todos estos estudios han sido realizados en centros de referencia con gran experiencia en ecoendoscopia y es posible que estos resultados no puedan generalizarse en la práctica clínica habitual al tratarse de una técnica muy dependiente del explorador.
El objetivo de nuestro estudio fue valorar: a) la utilidad de la ecoendoscopia en el estudio de la vía biliar extrahepática en los pacientes diagnosticados de pancreatitis aguda biliar en nuestro medio; y b) determinar los factores clínicos y analíticos relacionados con el resultado de la técnica que podrían ser utilizados para seleccionar los pacientes que más probablemente que se beneficiaran de su realización.

\section{MATERIAL Y MÉTODOS}

\section{Población de estudio}

Estudio realizado entre los pacientes remitidos a la Unidad de Endoscopias del Servicio de Aparato Digestivo de nuestro hospital, para la realización de ecoendoscopia biliopancreática, desde enero de 2005 a enero de 2007.

Incluimos en el estudio de modo consecutivo pacientes que presentaban datos clínicos y analíticos compatibles con el diagnóstico de pancreatitis aguda (valor de amilasa sérica al menos tres veces su valor normal) y en los que tras la realización de historia clínica, exploración física, estudios analíticos y ecografía abdominal habían sido diagnosticados de pancreatitis aguda biliar en base a la identificación ecográfica de colelitiasis y la ausencia de: a) trastornos metabólicos incluidos la hiperlipidemia o la hipercalcemia; b) antecedente reciente (en los tres últimos meses) de cirugía o traumatismo abdominal; c) toma reciente (en las dos últimas semanas) de tóxicos o fármacos relacionados con la pancreatitis aguda (16); d) no consumo previo de alcohol (> $80 \mathrm{~g} /$ día) en las dos semanas previas al episodio de pancreatitis; y e) ausencia de antecedentes familiares de pancreatitis o fibrosis quística.

Las variables clínicas y epidemiológicas recogidas en estos pacientes fueron: los datos demográficos; la severidad del episodio de pancreatitis aguda, según los criterios propuestos por Ranson y cols. (17), considerando pancreatitis aguda severa cuando se registraron tres o más criterios; y los parámetros analíticos al ingreso, incluidas enzimas pancreáticas, bilirrubina total y bioquímica hepática. Fueron excluidos los pacientes en los que se identificó por ecografía transabdominal la presencia de coledocolitiasis (siete pacientes), aquellos previamente colecistectomizados (tres pacientes), aquellos en los que no se pudo recoger una historia clínica adecuada (un paciente) y aquellos en los que no se pudo realizar (dos pacientes con gastrectomía Billroth II) o rechazaron la realización de la ecoendoscopia (un paciente). 


\section{Estudio ecoendoscópico}

El equipo endoscópico empleado fue un ecoendoscopio Olympus ${ }^{\circledast}$ modelo GF-UMQ130, utilizando frecuencias de 7,5 y $20 \mathrm{MHz}$. La exploración se realizó con el paciente en decúbito lateral izquierdo utilizando sedación consciente con midazolam o propofol. Las imágenes del páncreas, vesícula y vía biliar se obtuvieron colocando la punta del endoscopio en cuerpo gástrico, antro distal, bulbo y segunda porción duodenal. Se consideró como un resultado positivo el diagnóstico de coledocolitiasis que se hizo al observar en el interior de la vía biliar material ecogénico con o sin sombra acústica posterior (Figs. 1 y 2). Se recogieron datos como el calibre de la vía biliar, considerándola dilatada cuando presentaba un calibre mayor de $6 \mathrm{~mm}$, el tiempo transcurrido desde el ingreso hospitalario hasta la realización de la ecoendoscopia y, en caso de existir, el tamaño de las coledocolitiasis.

En la realización de los estudios participaron 2 exploradores con al menos 3 años de experiencia realizando ecoendoscopia (RGR y AR), en la misma unidad de trabajo y con el mismo método.

En todos los pacientes se obtuvo un consentimiento informado previo a la exploración endoscópica.

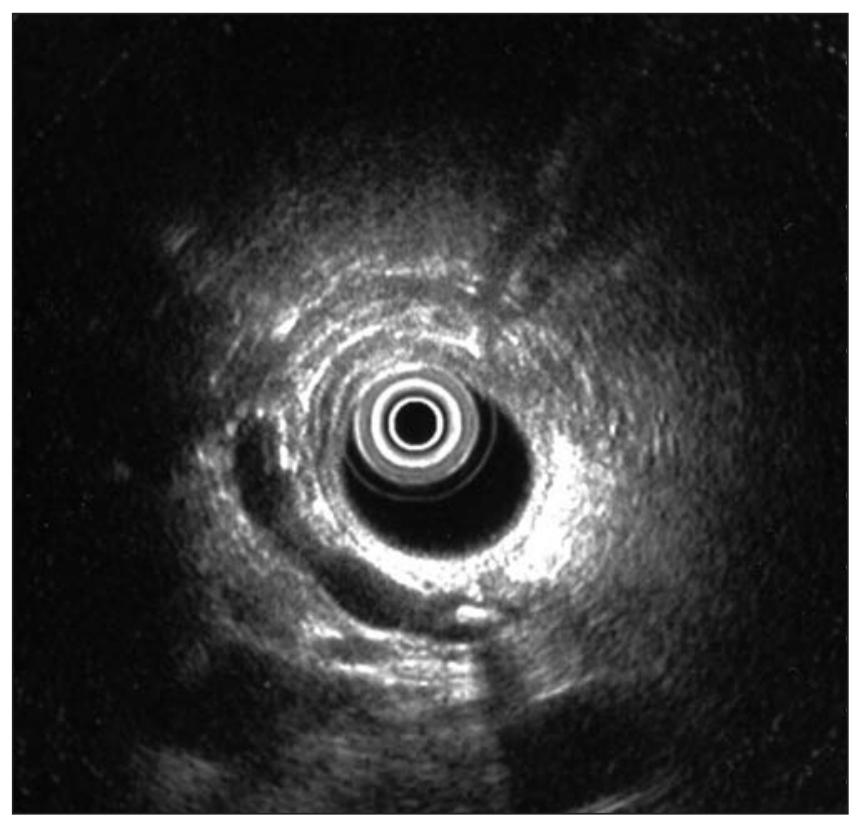

Fig. 1. Coledocolitiasis en forma de imagen hiperecogénica con sombra acústica a nivel papilar.

\section{Seguimiento}

De modo general, el enfoque terapéutico que se siguió en los pacientes diagnosticados de coledocolitiasis fue la realización de CPRE con colangiografía, esfinterotomía y técnica endoscópica para la extracción de cálculos. La ecoendoscopia y la CPRE no se realizaron en el mismo día. Sólo en los pacientes en los que se demostró endos-

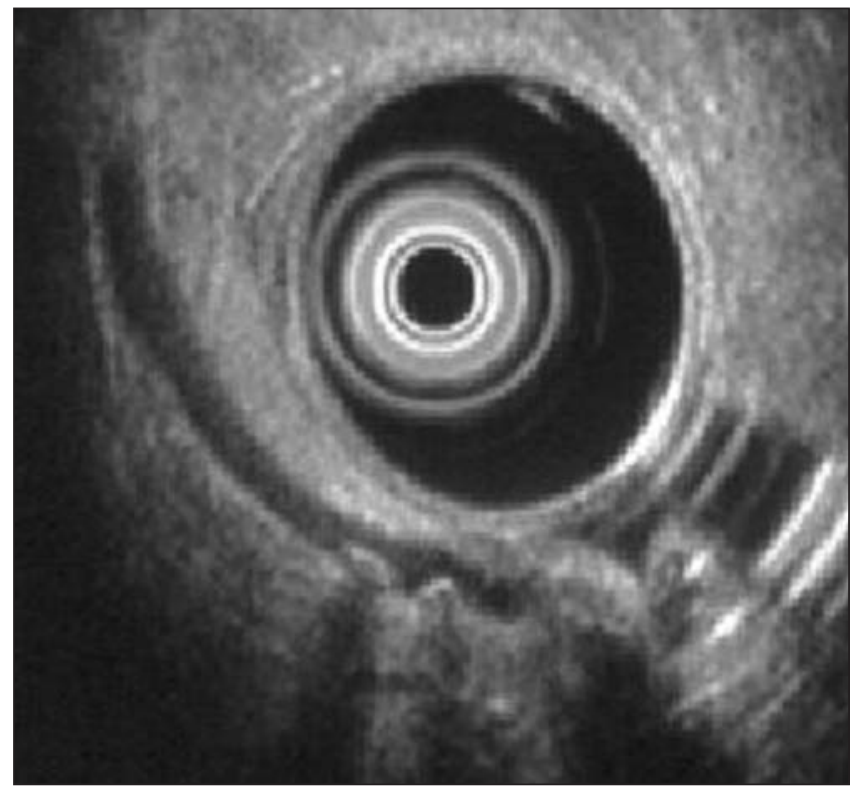

Fig. 2. Litiasis múltiples de pequeño tamaño $(3 \mathrm{~mm})$ con sombra acústica posterior en la porción distal de la vía biliar extrahepática.

cópicamente la salida de litiasis o fragmentos de estas se consideraron auténticas coledocolitiasis. Aquellos pacientes que no presentaban alto riego quirúrgico fueron intervenidos con realización de colecistectomía. La vesícula resecada fue inspeccionada para confirmar la existencia de litiasis y fue examinada histológicamente.

Todos los pacientes incluidos en el estudio fueron seguidos en régimen ambulatorio, con visitas cada tres o seis meses y ecografía abdominal cada seis o doce meses, en función de la evolución y sospecha diagnóstica. Se recogieron los datos relevantes de la evolución clínica, incluido el tiempo de seguimiento, el tiempo transcurrido desde la realización de la ecoendoscopia a la realización de la CPRE, el tiempo de estancia hospitalaria y la aparición de complicaciones locales o sistémicas.

\section{Análisis estadístico}

Las variables cuantitativas se indican con la media \pm desviación estándar. Para la comparación de porcentajes se utilizó la prueba de la Chi cuadrado y, cuando al menos más del $25 \%$ de los valores esperados eran menores de 5, mediante el test de Fisher. Se consideraron estadísticamente significativos valores de $\mathrm{p}$ menores de 0,05 .

\section{RESULTADOS}

Se incluyeron en el estudio 73 pacientes diagnosticados de pancreatitis aguda biliar, con una edad media de $64 \pm 15$ años, de los que 31 eran varones y 42 mujeres. El tiempo transcurrido desde el inicio de los síntomas hasta la realiza- 
ción de la exploración con ecoendoscopia fue de $7 \pm 6$ días. No se registraron complicaciones relacionadas con la técnica. En trece pacientes (18\%) se registraron más de tres criterios de Ranson. En todos ellos la ecoendoscopia se realizó en los tres primeros días del ingreso hospitalario.

Se consiguió obtener por ecoendoscopia imágenes de la vía biliar extrahepática en todos los pacientes y en 18 (24\%) se encontraba dilatada. En 18 (24\%) se observó la existencia de coledocolitiasis. El tamaño medio de estas litiasis era de 4,8 $\mathrm{mm}$ (rango de 2 a $11 \mathrm{~mm}$ ).

Se intentó realizar CPRE con esfinterotomía endoscópica en 19 pacientes, incluido un paciente de alto riesgo quirúrgico en el que no se había descrito por ecoendoscopia la presencia de coledocolitiasis y se planteó la esfinterotomía endoscópica como tratamiento definitivo (Fig. 3 ). Se consiguió cateterizar la vía biliar en todos menos en un paciente, en el que finalmente se decidió explorar quirúrgicamente la vía biliar, confirmándose la presencia de coledocolitiasis en la colangiografía intraoperatoria. El tiempo medio transcurrido desde la realización de la ecoendoscopia a la CPRE fue de $5 \pm 4$ días.

De las 18 CPRE finalmente realizadas se observó endoscópicamente la presencia de coledocolitiasis en todos menos en dos pacientes: el paciente en el que se realizó CPRE a pesar del resultado negativo de la ecoendoscopia y otro en el que la CPRE se demoró 9 días. Se registró una complicación derivada de una esfinterotomía endoscópica (hemorragia postesfinterotomía controlada con esclerosis endoscópica).

Cuando se analizaron los factores clínicos y analíticos relacionados con el resultado de la ecoendoscopia, observamos cómo la presencia de coledocolitiasis fue más frecuente en aquellos pacientes con dilatación de la vía biliar extrahepática (55 vs. 14\%; p < 0,05) y en aquellos con bilirrubina sérica superior a $3 \mathrm{mg} / \mathrm{dl}(41 \mathrm{vs} .18 \%$; p < $0,05)$. Así mismo, en aquellos pacientes con dilatación de

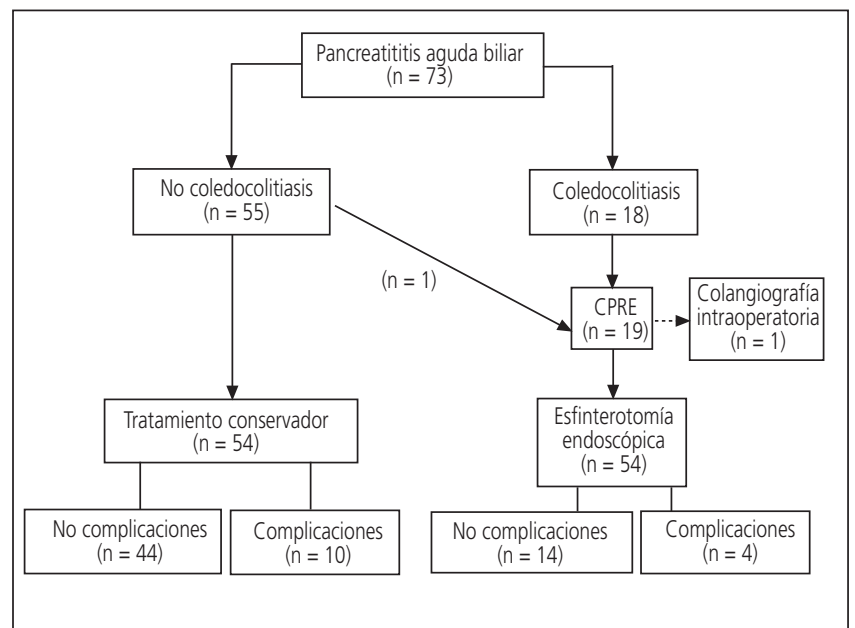

Fig. 3. Resultados del estudio de la vía biliar por ecoendoscopia, tratamiento endoscópico y complicaciones durante el ingreso hospitalario.

la vía biliar y bilirrubina mayor de $3 \mathrm{mg} / \mathrm{dl}$, la probabilidad de presentar coledocolitiasis fue del $75 \%$. No se observó relación significativa entre la demora en la realización de la USE superior a dos días y la presencia de coledocolitiasis (Tabla I).

Catorce pacientes (19\%) presentaron alguna complicación durante el ingreso en relación directa o indirecta con la pancreatitis aguda, incluidas 10 complicaciones sistémicas (dos pacientes con insuficiencia respiratoria, dos con insuficiencia renal, una neumonía nosocomial, un flutter auricular, una hemorragia digestiva, dos diarreas por antibióticos y una colitis pseudomembranosa) y 4 complicaciones locales (dos colangitis agudas, un absceso pancreático y un pseudoquiste pancreático). Un paciente murió en relación con fallo multiorgánico. No se observó diferencia significativa en el porcentaje de com-

Tabla I. Características clínicas y analíticas de 73 pacientes con pancreatitis aguda biliar en los que se estudió por ecoendoscopia la vía biliar extrahepática

\begin{tabular}{|c|c|c|c|c|}
\hline & $\begin{array}{l}\text { Número de } \\
\text { pacientes }\end{array}$ & $\begin{array}{l}\text { Presencia de } \\
\text { coledocolitiasis }\end{array}$ & $\begin{array}{l}\text { Ausencia de } \\
\text { coledocolitiasis }\end{array}$ & Valor de $p$ \\
\hline Edad ( $\leq 50$ años) & 22 & $3(14 \%)$ & $19(86 \%)$ & NS \\
\hline Sexo: Femenino & 42 & $11(26 \%)$ & $31(73 \%)$ & NS \\
\hline Masculino & 31 & $7(22 \%)$ & $24(77 \%)$ & NS \\
\hline Pancreatitis severa & 13 & $2(15 \%)$ & $11(85 \%)$ & NS \\
\hline $\begin{array}{l}\text { Más de } 2 \text { días desde el inicio de los sín } \\
\text { de la ecoendoscopia }\end{array}$ & 62 & $16(26 \%)$ & 46 (74\%) & NS \\
\hline Dilatación de vía biliar (> 6 mm) & 18 & $10(55 \%)$ & $45(14 \%)$ & $<0,05$ \\
\hline Amilasa > $500 \mathrm{mU} / \mathrm{ml}$ & 54 & $14(26 \%)$ & $40(74 \%)$ & NS \\
\hline Bilirrubina $>3 \mathrm{mg} / 100 \mathrm{ml}$ & 22 & $9(41 \%)$ & $13(59 \%)$ & $<0,05$ \\
\hline $\mathrm{GOT}>40 \mathrm{mU} / \mathrm{ml}$ & 56 & $14(25 \%)$ & $42(75 \%)$ & NS \\
\hline GPT > $40 \mathrm{mU} / \mathrm{ml}$ & 61 & $15(24 \%)$ & $46(76 \%)$ & NS \\
\hline $\mathrm{GGT}>50 \mathrm{mU} / \mathrm{ml}$ & 67 & $17(25 \%)$ & $50(75 \%)$ & NS \\
\hline Fosfatasa alcalina > $130 \mathrm{mU} / \mathrm{ml}$ & 37 & $11(30 \%)$ & $26(70 \%)$ & NS \\
\hline $\begin{array}{l}\text { Bilirrubina > } 3 \mathrm{mg} / 100 \mathrm{ml} \text { y dilatación } \\
\text { de vía biliar }\end{array}$ & 8 & $6(75 \%)$ & $2(25 \%)$ & $<0,05$ \\
\hline
\end{tabular}


plicaciones en los pacientes tratados de modo conservador respecto a aquellos con coledocolitiasis tratados con esfinterotomía endoscópica (18 vs. 22\%; p > 0,05). Tampoco se observó esta diferencia en el subgrupo de pacientes con pancreatitis aguda severa (50 vs. 45\%; p >0,05).

Durante un tiempo de seguimiento medio de $12 \pm 5$ meses tres pacientes presentaron nuevos episodios de pancreatitis aguda. En todos estos casos se trataba de pacientes en los que no se había realizado CPRE, no se describía la existencia de coledocolitiasis en la ecoendoscopia y en todos los casos antes de la realización de la colecistectomía.

En base a estos resultados, la sensibilidad de la ecoendoscopia para el diagnóstico de coledocolitiasis en pacientes con pancreatitis aguda biliar fue del $85 \%$, la especificidad del $98 \%$, el valor predictivo positivo del $94 \%$, el valor predictivo negativo del $94 \%$ y la exactitud diagnóstica del $94 \%$.

\section{DISCUSIÓN}

La ecoendoscopia, al utilizar un haz de ultrasonidos de alta frecuencia y evitar los artefactos aéreos que limitan la exploración del sistema biliar en la ecografía transabdominal, permite obtener imágenes de alta calidad del sistema biliar, por lo que ha sido utilizada con un alto rendimiento en la detección de coledocolitiasis (18). En los estudios realizados a este respecto se describe una sensibilidad diagnóstica del $93 \%$, especificidad del $96 \%$, valor predictivo positivo del $93 \%$ y valor predictivo negativo del $96 \%$ para el diagnóstico de coledocolitiasis (19). Sin embargo, en el grupo de pacientes con pancreatitis aguda la exploración por ecoendoscopia de la vía biliar podría resultar más compleja por las alteraciones parenquimatosas, la presencia de necrosis y/o colecciones líquidas que impiden una correcta valoración del colédoco intrapancreático (11). Además sigue siendo controvertido cuál ha de ser el mejor manejo endoscópico en los pacientes con pancreatitis aguda biliar (20).

La utilidad de la ecoendoscopia en el estudio de la vía biliar extrahepática en pacientes con pancreatitis aguda biliar ha sido previamente evaluada de forma retrospectiva por Prat y cols. en 123 pacientes en los que se hizo ecoendoscopia seguida de CPRE terapéutica en la misma sesión endoscópica cuando se observó la presencia de coledocolitiasis (11). En todos los casos en los que se identificó ecográficamente la presencia de litiasis se consiguió cateterizar por CPRE la vía biliar, la realización de esfinterotomía endoscópica y se comprobó endoscópicamente su presencia. Ninguno de los pacientes con pancreatitis severa y un resultado negativo de la ecoendoscopia presentó recurrencia de los síntomas biliares durante el seguimiento. Concluyen los autores que en estos pacientes podría plantearse la realización selectiva de la esfinterotomía endoscópica en función del resultado de la ecoendoscopia.

El resultado de otras series prospectivas también apoya la utilización de la ecoendoscopia en estos pacientes (12-
14) y su eficacia y seguridad en esta indicación fue confirmada en un estudio prospectivo en el que se aleatorizó a 140 pacientes con pancreatitis aguda biliar a la realización de CPRE (70 pacientes) o ecoendoscopia (70 pacientes), seguido de CPRE terapéutica en caso de observar coledocolitiasis (15). No se observaron diferencias significativas en la tasa total de complicaciones (7\% en el grupo de la ecoendoscopia y $14 \%$ en el grupo de CPRE), la estancia hospitalaria ni la mortalidad en ambos grupos.

A los argumentos clínicos se suman los económicos y en un análisis teórico de coste-efectividad en el que se compara la realización de ecoendoscopia seguida de CPRE (en la misma sesión) sólo en los casos en los que se identifica la existencia de coledocolitiasis con la denominada "pauta de actuación habitual", que consiste en realizar CPRE en todos los pacientes con pancreatitis aguda biliar severa y en aquellos con pancreatitis aguda biliar no severa en función de la persistencia de la ictericia y/o la dilatación de la vía biliar (21). Se describe cómo la realización de la pauta con ecoendoscopia resulta más barata que la "pauta habitual", debido a un menor número de complicaciones relacionadas con la CPRE.

La presencia de coledocolitiasis se describe en el 27$36 \%(11,13)$ de los pacientes con pancreatitis aguda biliar. En nuestra serie se identificó la presencia de coledocolitiasis en el $24 \%$ de los pacientes. Sin embargo, debido a la demora de $7 \pm 6$ días desde el inicio de los síntomas hasta la realización de la exploración, es posible que en algún caso se produjese la migración espontánea de la litiasis. A este respecto, en alguna serie se describe cómo la proporción de litiasis residual identificadas fue mayor cuando la ecoendoscopia se realizó en los tres primeros días desde la presentación de los síntomas en comparación con la ecoendoscopia trascurridos más de tres días (11) e incluso se ha descrito cómo hasta en el $20 \%$ de los pacientes con coledocolitaisis menores de $8 \mathrm{~mm}$ se produce su migración espontánea, a menudo en ausencia de síntomas, transcurridas de 6 horas a 1 mes desde su identificación por ecoendoscopia, siendo esta proporción aun mayor en pacientes con pancreatitis aguda (22). Por esta misma razón y debido a la demora en la realización de la CPRE en nuestros pacientes (media de $5 \pm 4$ días), es posible que en un caso en el que se observó la presencia de coledocolitiasis en la ecoendoscopia no se confirmase en la CPRE posterior. El procedimiento ideal probablemente sea la realización de la ecoendoscopia y CPRE en la misma sesión endoscópica (11). Sin embargo, por razones logísticas esto no es posible en la mayoría de los centros. En el futuro, incluso podría realizarse la exploración ecográfica, colangiográfica y terapéutica con un mismo endoscopio actualmente en desarrollo (23).

En las distintas series se describe una tasa de resultados falsos negativos variable del 0 al $9 \%$, que se ha relacionado con las dificultades técnicas en el estudio por ecoendoscopia de la vía biliar extrahepática en pacientes con pancreatitis aguda y en ocasiones con el pequeño tamaño de las microlitiasis en la vía biliar implicados en el desarrollo 
de la inflamación pancreática (11-13) (Fig. 2). En todos los pacientes de nuestra serie que presentaron nuevos episodios de pancreatitis aguda durante el seguimiento, estos ocurrieron transcurridas al menos 4 semanas y antes de la realización de la colecistectomía, por lo que más que errores de la técnica podrían ser debidos a una demora excesiva en la realización de la colecistectomía.

Cuando se han analizado los factores relacionados con la presencia de coledocolitiasis en pacientes con colelitiasis previa a la realización de colecistectomía, se describe cómo en aquellos que presentan dilatación de la vía biliar y elevación de enzimas de colestasis el riesgo de presentar coledocolitiasis es del 50-80\%. Por lo que algunos autores recomiendan en estos casos la realización de una colecistectomía acompañada de exploración quirúrgica de la vía biliar o CPRE con esfinterotomía endoscópica previa a la colecistectomía en función de la experiencia y disponibilidad en cada centro $(24,25)$. Aun en estos casos con "alto riesgo" de presentar coledocolitiasis, cuando han transcurrido varios días desde el inicio de los síntomas y puede haberse producido la eliminación espontánea de la litiasis, podría plantearse la realización de una ecoendoscopia para verificar la presencia de coledocolitiasis (25). En los pacientes con pancreatitis aguda biliar de nuestra serie observamos resultados similares en el estudio de la vía biliar extrahepática.

No ha sido claramente establecido si la esfinterotomía endoscópica en pacientes con pancreatitis aguda biliar en ausencia de coledocolitiasis puede influir positivamente en la evolución de estos pacientes (20). La respuesta a esta cuestión se habrá de basar en el resultado de estudios en los que se aleatorice a estos pacientes a la realización de esfinterotomía endoscópica o tratamiento conservador. Aunque nuestro estudio no fue diseñado a este respecto, no observamos diferencia en el porcentaje de complicaciones en los pacientes tratados de modo conservador respecto a aquellos con coledocolitiasis tratados con esfinterotomía endoscópica. Además de estas cuestiones, no claramente establecidas, la decisión de realizar una esfinterotomía en estos pacientes en ausencia de coledocolitiasis deberá individualizarse en función de otros factores como el riesgo quirúrgico y la demora en la realización de la colecistectomía (2).

En conclusión, la ecoendoscopia es una técnica útil en la selección de los pacientes con pancreatitis aguda biliar que se beneficiarán de la realización de una esfinterotomía endoscópica.

\section{BIBLIOGRAFÍA}

1. Carballo Álvarez F, Mateos Hernández J. Pancreatitis aguda: Epidemiología y etiología. En: Navarro S, Pérez-Mateo M y Guarner L, editores. Tratado de páncreas exocrino. Barcelona: J\&C Ediciones Médicas S.L.; 2002. p. 118-33.

2. Pandol SJ, Saluja AK, Imrie CW, Banks PA. Acute pancreatitis: Bench to the bedside. Gastroenterology 2007; 132: 1127-51.

3. Neoptolemos JP, London NJ, James D, Carr-Locke DL, Bailey IA,
Fossard DP. Controlled trial of urgent endoscopic retrograde cholangiopancreatography and endoscopic sphincterotomy versus conservative treatment for acute pancreatitis due to gallstones. Lancet 1988; 2: 979-83.

4. Fan ST, Lai ECS, Mok FPT, Lo CM, Zheng SS, Wong J. Early treatment of acute biliary pancreatitis by endoscopic papillotomy. N Engl J Med 1993; 328: 228-32.

5. Fölsch UR, Nitsche R, Lüdtke R, Hilgers RA, Creutzfeldt W. Early ERCP and papillotomy compared with conservative treatment for acute biliary pancreatitis. The German Study Group on Acute Biliary Pancreatitis. N Engl J Med 1997; 336 (4): 237-42.

6. Freeman ML, Nelson DB, Sherman S, Haber ME, Dorsher PJ, Moore JO, et al. Complications of endoscopic biliary sphincterotomy. N Engl J Med 1996; 335: 909-18.

7. Priego P, Rodríguez G, Mena A, Losa N, Aguilera A, Ramiro C, et al. Hematoma subcapsular hepático tras CPRE. Rev Esp Enferm Digest 2007; 99: 49-50.

8. García-Martínez MT, Ruano A, Galán L, Gay AM, Casal JE. Neumoperitoneo tras colangiografía retrógrada endoscópica. Rev Esp Enferm Digest 2007; 99: 421-2.

9. Amouyal P, Palazzo L, Amouyal G, Ponsot P, Mompoint D, Vilgrain $\mathrm{V}$, et al. Endosonography: Promising method for diagnosis of extrahepatic cholestasis. Lancet 1989; 18: 1195-8.

10. Dancygier H, Nattermann C. The role of endoscopic ultrasonography in biliary tract disease: Obstructive jaundice. Endoscopy 1994; 26: 800-2.

11. Prat F, Edery J, Meduri B, Chiche R, Ayoun C, Bodart M, et al. Early EUS of the bile duct before endoscopic sphincterotomy for acute biliary pancreatitis. Gastrointest Endosc 2001; 54: 724-9.

12. Chak A, Hawes RH, Cooper GS, Hoffman B, Catalano MF, Wong $\mathrm{RC}$, et al. Prospective assessment of the utility of EUS in the evaluation of gallstone pancreatitis. Gastrointest Endosc 1999; 49: 599-604.

13. Sugiyama M, Atomy Y. Acute biliary pancreatitis: The roles of endoscopic ultrasonography and endoscopic retrograde cholangiopancreatography. Surgery 1998; 124: 14-21.

14. Liu CL, Lo CM, Chan JKF, Poon RT, Lam CM, Fan ST, et al. Detection of choledocholithiasis by EUS in acute pancreatitis: A prospective evaluation in 100 consecutive patients. Gastrointest Endosc 2001; 54: 325-30.

15. Liu CL, Fan ST, Lo CM, Tso WK, Wong Y, Poon RT, et al. Comparison of early endoscopic ultrasonography and endoscopic retrograde cholangiopancreatography in the management of acute pancreatitis: A prospective randomized study. Clin Gastroenterol Hepatol 2005; 3: $1238-44$.

16. Somogyl L, Martin SP, Venkatesan T, Ulrich CD. Recurrent acute pancreatitis: An algorithmic approach to identification and elimination of inciting factors. Gastroenterology 2001; 120: 708-17.

17. Ranson JHC, Rifkind KM, Roses DF, Fink SD, Eng K, Spencer FC. Prognostic signs and the role of operative management in acute pancreatitis. Surg Gynecol Obstet 1974; 139: 69-81.

18. Napoleon B, Lefort C, Gincoul R. State of the art lecture: Lithiasis and pancreatitis. Endoscopy 2006; 38: S35-S40.

19. Verma D, Kapadia A, Eisen GM, Adler DG. EUS vs. MRCP for detection of choledocholithiasis. Gastrointest Endosc 2006; 64: 248-54.

20. Petrov MS. ERCP in acute biliary pancreatitis: The moor has done his duty, the moor may go. Am J Gastroenterol 2007; 102: 2859-60.

21. Romagnuolo J, Currie G, the Calgary Advanced Therapeutic Endoscopy Center (ATEC) study group. Non-invasive vs. selective invasive biliary imaging for acute biliary pancreatitis: An economic evaluation by using decision tree analysis. Gastrointest Endosc 2005; 61: 86-97.

22. Frossard JL, Hadengue A, Amouyal G, Choury A, Marty O, Giostra E, et al. Choledocholithiasis: A prospective study of spontaneous common bile duct stone migration. Gastrointest Endosc 2000; 51 : $175-9$.

23. Rocca R, De Angelis C, Castellino F, Masoero G, Daperno M, Sostegni $\mathrm{R}$, et al. EUS diagnosis and simultaneous endoscopic retrograde cholangiography treatment of common bile duct stones by using an obliqueviewing echoendoscope. Gastrointest Endosc 2006; 63: 479-84.

24. Napoleón B. Ecoendoscopia y patología biliar. Gastroenterol Hepatol 2002; 25: 35-41.

25. O'Toole D, Palazzo L. Choledocholithiasis -a practical approach from the endosonographer. Endoscopy 2006; 38: S23-9. 